\title{
Uso de estomas sintéticos en carcinomatosis abdominal. Informe de caso y revisión de la bibliografía
}

\section{Use of synthetic stomata in abdominal carcinomatosis. Case report and literature review}

Federico Mendoza-Sánchez ${ }^{*}$, Wenceslao G. Ángeles-Bueno ${ }^{1}$, Gustavo A. Montes-López', Diego F. Mendoza-Medina ${ }^{1}$, José M. Díaz-Sifuentes ${ }^{2}$ y Jacob E. Pérez-Landeros ${ }^{2}$

${ }^{1}$ Departamento de Cirugía, Hospital San Javier; ${ }^{2}$ Unidad de Investigación Biomédica 02, Hospital de Especialidades, Centro Médico Nacional de Occidente, Instituto Mexicano del Seguro Social. Guadalajara, Jalisco, México

\begin{abstract}
Resumen
Introducción: Desde el año 1706 se han descrito ostomías realizadas con éxito para el tratamiento de diferentes enfermedades; los autores describen el primer caso de éxito en una ostomía sintética en la carcinomatosis peritoneal. Caso clínico: Mujer de 40 años de edad con carcinomatosis abdominal por adenocarcinoma papilar tubulopapilar psamomatoso más consistente con cáncer primario de ovario, negativo a receptores de estrógenos y progesterona, con marcador Ki-67 al $83 \%$ de actividad. De modo inicial se trató con cirugía de citorreducción, quimioterapia, quimioterapia intraperitoneal hipertérmica y por último realización de estomas sintéticos debido a perforaciones entéricas e imposibilidad de realizar estomas descompresivos autólogos por la invasión tumoral extensa y adherencias de todas las asas intestinales. Conclusiones: Los estomas sintéticos pueden ser una buena opción terapéutica cuando es imposible realizar estomas autólogos.
\end{abstract}

PALABRAS CLAVE: Estomas sintéticos. Adenocarcinoma de ovario. Citorreducción. HIPEC.

\begin{abstract}
Background: The successful performance of ostomies for the treatment of different diseases has been described since 1706. We report herein the first case of successful ostomy utilizing a synthetic stoma created in a patient with peritoneal carcinomatosis. Clinical case: A 40-year-old woman presented with abdominal carcinomatosis due to psammomatous papillotubular adenocarcinoma consistent with primary ovarian carcinoma. The patient had negative estrogen and progesterone receptors and Ki-67 proliferative activity was $83 \%$. She was initially treated with cytoreduction therapy, chemotherapy, and hyperthermic intraperitoneal chemotherapy. Because the patient presented with enteric perforations and the extensive tumor invasion and adhesions in all the intestinal segments made it impossible to create autologous decompression stomas, a synthetic stoma was constructed. Conclusions: Synthetic stomas can be a good treatment option when autologous stomas can not be created.
\end{abstract}

KEY WORDS: Synthetic stomata. Adenocarcinoma of ovary. Cytoreduction. HIPEC.

\footnotetext{
Correspondencia:

*Federico Mendoza-Sánchez

Avda. Puerta de Hierro, 5150, 303C

Col. Plaza Corporativa Zapopan

C.P. 45116, Zapopan, Jalisco, México

E-mail: federico_mendozas1@ hotmail.com
}

Fecha de recepción: 16-07-2018

Fecha de aceptación: 06-04-2019

DOI: 10.24875/CIRU.19000605
Cir Cir. 2019;87(S1):28-32

Contents available at PubMed www.cirugiaycirujanos.com 


\section{Introducción}

Praxágoras de Cos (384-322 a. C.) describió la formación de una fístula intestinal, con lo cual inició el tratamiento quirúrgico de la obstrucción. Sólo hasta el año 1706 se documentó una colostomía practicada en el soldado George Deppe, quien sufrió una herida abdominal y sobrevivió más de 14 años con la colostomía prolapsada. Cuatro años después, en 1710, Littre de París recomendó la práctica sistemática de la cecostomía inguinal transperitoneal en todos los pacientes con ano imperforado ${ }^{1-3}$; desde entonces se han efectuado diferentes tipos de estomas para múltiples enfermedades, pero hasta ahora no se ha notificado ninguna operación de estomas con material sintético en el medio de los autores; es el tratamiento que se describe a continuación.

\section{Caso clínico}

A principios de noviembre del 2015, una mujer de 40 años se presentó por dolor abdominal intenso y difuso de inicio súbito, acompañado de distensión abdominal. No tenía antecedentes médicos, familiares o quirúrgicos de relevancia.

Dos días después de su ingreso fue objeto de intervención quirúrgica; se realizó drenaje y citología del líquido ascítico y se diagnosticó ascitis por carcinomatosis abdominal originada por adenocarcinoma, más consistente con un cáncer primario de ovario. Se practicó histerectomía, salpingooforectomía bilateral, apendicectomía, omentectomía, cirugía citorreductiva (CCR) y tumorectomías múltiples de la raíz y porciones antimesentéricas de todo el intestino (Fig. 1). Se observó una buena evolución posquirúrgica, por lo que recibió el alta médica siete días después.

A los 20 días de la intervención se llevó a cabo control de antígeno CA-125 (2 $480 \mathrm{U} / \mathrm{ml})$ y se obtuvo el resultado de un estudio histopatológico: adenocarcinoma papilar tubulopapilar psamomatoso más consistente con primario de ovario, negativo a receptores de estrógenos y progesterona, con marcador Ki-67 a $83 \%$ de actividad.

Se administraron paclitaxel más carboxiplatino (6 ciclos); a continuación carboplatino más doxorrubicina liposomal y bevacizumab (3 ciclos) y al final docetaxel (monoterapia), fármaco con el que se continuó.

En su evolución, el antígeno CA-125 decreció hasta $49.94 \mathrm{U} / \mathrm{ml}$; no obstante, se incrementó de nueva

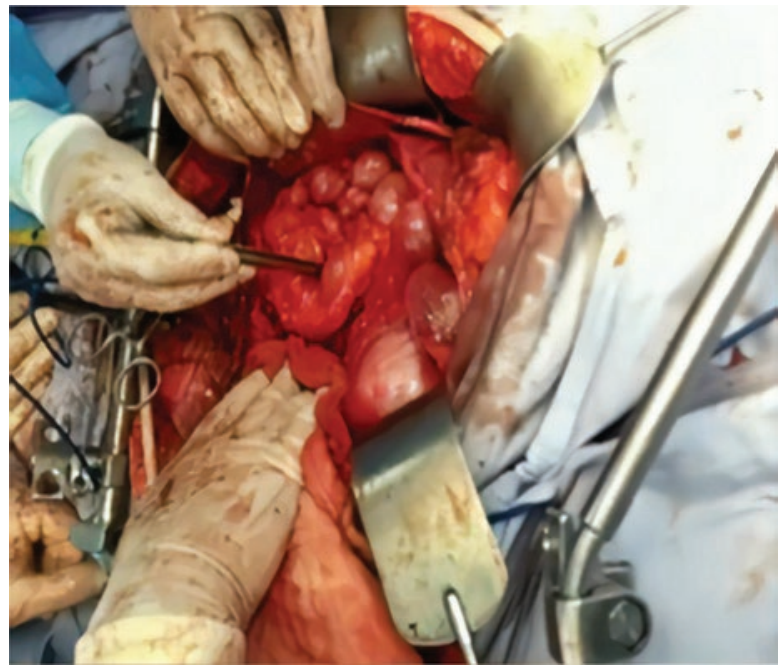

Figura 1. Cirugía de citorreducción.

cuenta a $93.3 \mathrm{U} / \mathrm{ml}$; debido a ello, en mayo de 2016 se decidió realizar peritonectomía, CCR, omentectomía total (del menor y mayor), esplenectomía, colecistectomía y electrocoagulación con argón plasma de toda la capsula hepática, además de quimioterapia intraperitoneal hipertérmica (HIPEC) con mitomicina C y colocación de malla Ethicon Physiomesh ${ }^{\circledR}$ (dispositivo de malla compuesta flexible abierta).

Cuatro meses después, la paciente presentó obstrucción intestinal parcial y signos de celulitis en la parte anterosuperior del miembro pélvico derecho, motivo por el cual se efectuó laparotomía exploradora, con lisis de bridas y adherencias accesibles. Se identificó una perforación de la cara posterior del ciego, que se reparó con Prolene ${ }^{\circledR}$ vascular 3-0 de doble armado (se drenó líquido fecal seropurulento intraabdominal, 3 $000 \mathrm{ml}$ ), y otra de la corredera parietocólica derecha a la parte anterosuperior del miembro pélvico derecho.

Ocho días después se reconocieron perforaciones de asas de yeyuno e íleon (de causa obstructiva tumoral maligna, múltiple y extensa) y una reapertura de la perforación del ciego; dado que era imposible realizar colostomía descompresiva por la invasión tumoral extensa y las adherencias de las asas intestinales, se colocaron estomas sintéticos. Se usaron injertos vasculares y prótesis de dacrón (Maquet ${ }^{\circledR}$ ) precoagulado con plata (Intergard silver ${ }^{\circledR}$ ) e impregnado con rifampicina (Fig. 2). Los injertos sintéticos vasculares se anastomosaron a los orificios enterales con Prolene ${ }^{\circledR}$ vascular 3-0 y 4-0.

Una semana después de la intervención se drenó abundante material seropurulento fecal-hemático en toda la porción retroperitoneal por encima de la vena 


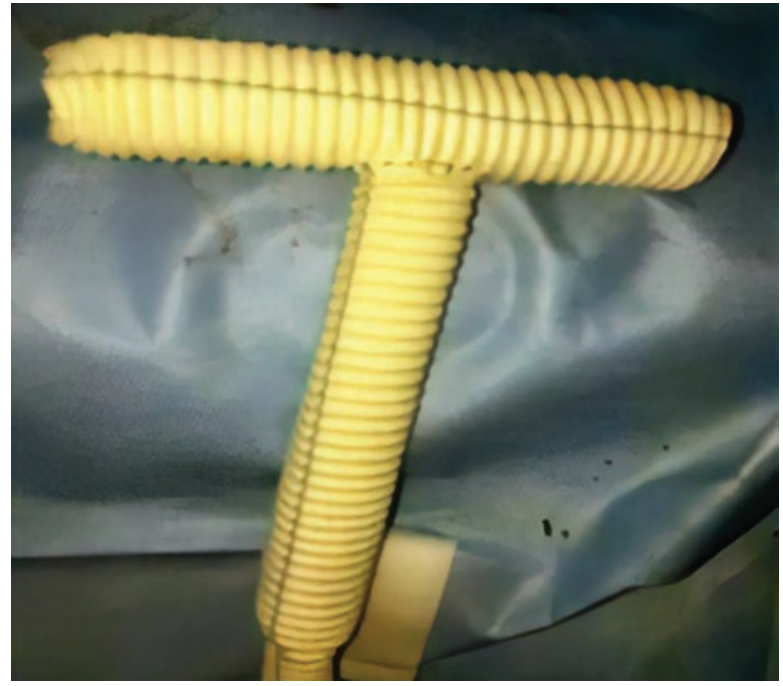

Figura 2. Injerto de dacrón precoagulado con plata e impregnado con rifampicina.

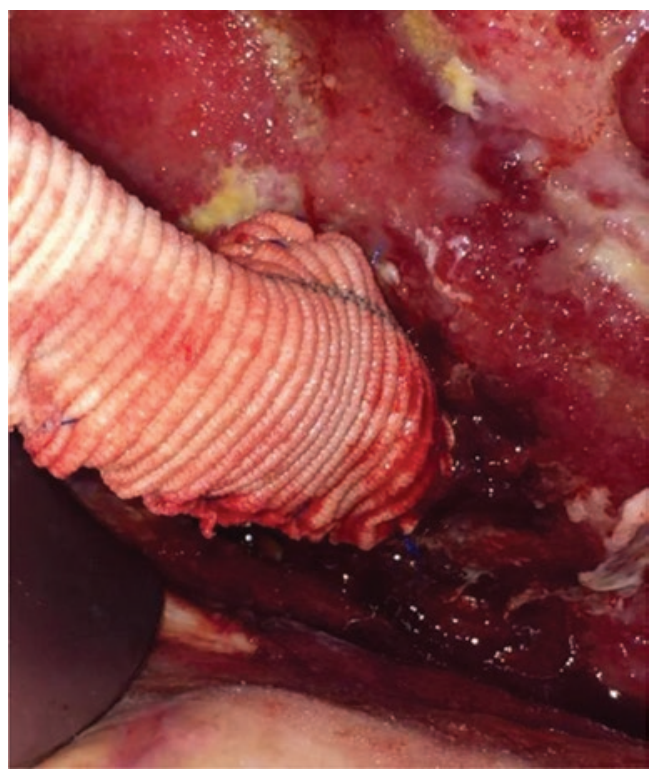

Figura 3. Colocación del estoma sintético en la perforación retrocólica del ciego.

cava, aorta y perirrenal bilateral. En las siguientes intervenciones se construyeron más ostomías sintéticas y se utilizaron también injertos sintéticos de dacrón precoagulado con plata e impregnados con rifampicina en las zonas de la perforación retrocólica del ciego (Figs. 3 y 4 ) y en las perforaciones yeyunales e ileales.

Se realizó un total de 22 operaciones abdominales y asepsias quirúrgicas. Se emplearon diversos materiales y técnicas quirúrgicas, de acuerdo con los hallazgos, para controlar los efectos secundarios de las perforaciones enterales. Se recurrió al sistema VAC (vacuum assisted closure) de aspiración continua

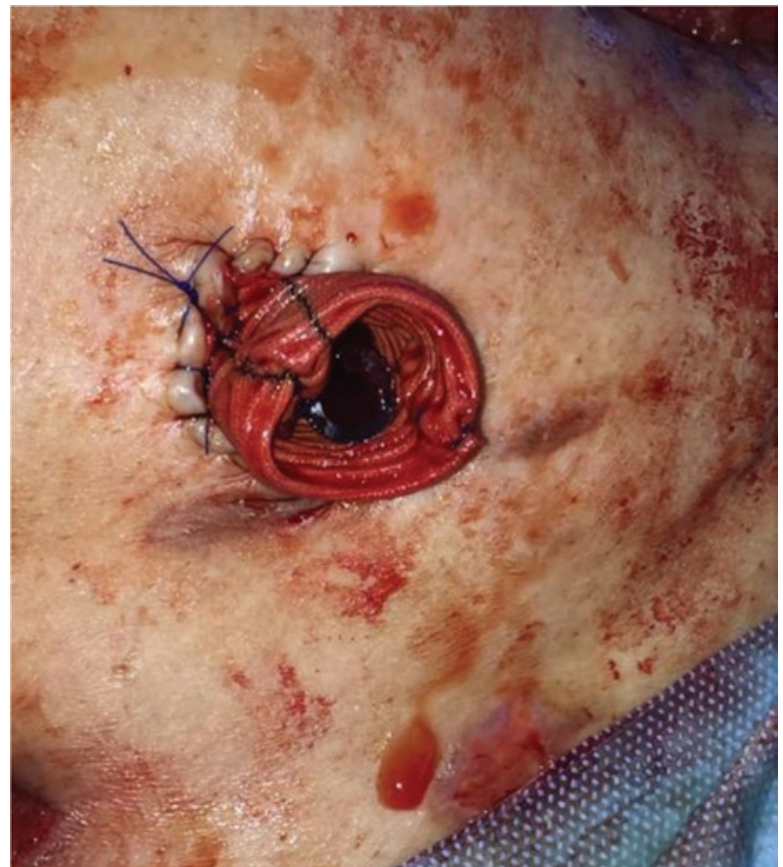

Figura 4. Colocación del estoma sintético bifurcado en las perforaciones yeyunales e ileales.

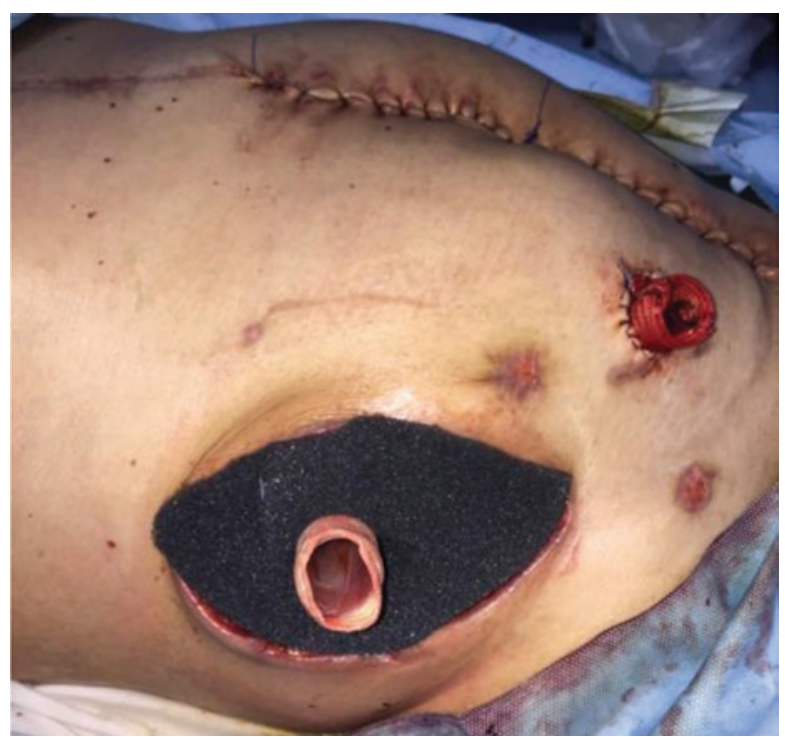

Figura 5. Utilización de sistema VAC y colocación del estoma sintético.

intermedia (Fig. 5), Gelfoam ${ }^{\circledR}$, Tissucol ${ }^{\circledR}$ (fibrinógeno, solución de fibrinógeno humano liofilizado, Baxter), Drawtex $^{\circledR}$ (hydroconductive wound dressing with LevaFiber $^{T M}$ technology), Oasis ${ }^{\circledR}$ (láminas de submucosa obtenida del intestino delgado de cerdo), apósitos de Garacoll$^{\circledR}$ (implante absorbible de colágeno con gentamicina) y Karaya ${ }^{\circledR}$ (caucho natural).

En diciembre del 2016, la paciente presentó broncoaspiración por invasión tumoral al estómago y duodeno (obstrucción gastroduodenointestinal) y a 


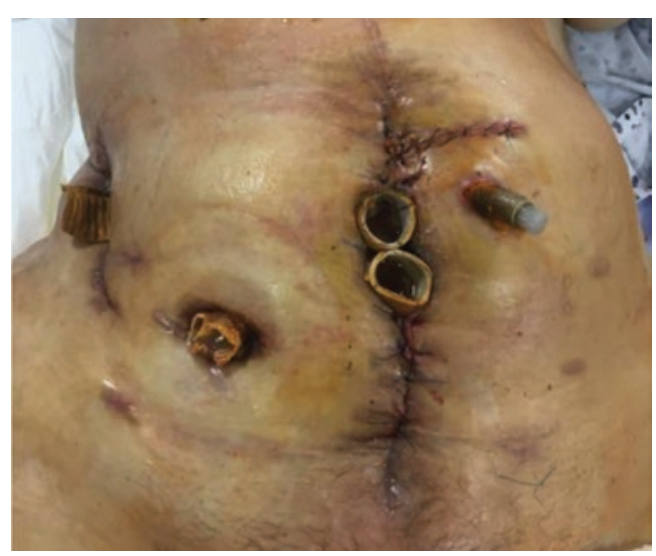

Figura 6. Estoma sintético ferulizado y con adecuado trayecto fistuloso.

continuación un absceso pulmonar izquierdo; se realizaron aspiraciones por broncoscopia, drenaje del absceso, pleurodesis y antibioticoterapia. Además, aparecieron infecciones abdominales (Escherichia coli y Enterococcus faecium), pulmonares (Escherichia coli portadora de ESBL, Candida glabrata), urinarias (Candida krusei), celulitis y fascitis (Escherichia coli y Enterococcus faecium), con hemocultivos positivos para Escherichia coli. Se controlaron las infecciones, se ferulizaron los estomas sintéticos y se fistulizaron de forma adecuada las perforaciones enterales (Fig. 6).

En enero de 2017 se elevó de nueva cuenta la cifra de Ag CA-125 a $3930 \mathrm{U} / \mathrm{ml}$, por lo cual se volvió a administrar quimioterapia. Por último, al cabo de 27 meses la paciente falleció por carcinomatosis y complicaciones agregadas de la quimioterapia (hipotensión, íleo paralítico y aplasia medular grave).

\section{Discusión}

La evolución de las ostomías desempeña un papel crucial en la terapéutica quirúrgica; éstas se utilizan con tres propósitos: descompresivo, desfuncionalizante y derivativo. Las ostomías descompresivas se indican cuando el intestino delgado o el colon presentan una dilatación excesiva y por ende con riesgo de fistulizarse o perforarse; su finalidad es liberar la presión intraluminal por medio de orificios (yeyunostomía, ileostomía, colostomía, sigmoidostomía, etc.) o la colocación de catéteres, ya sea en el estómago (gastrostomía) o el ciego (cecostomía). En fecha reciente se han agregado las colonoscopias descompresoras y el paso de catéteres o sondas a través del recto'.

El uso de materiales sintéticos (en particular sondas y tubos de drenaje) con el fin de descomprimir la obstrucción intestinal comenzó desde el decenio de 1980 al llevar a cabo gastrostomías y cecostomías con tal finalidad; en la actualidad se han convertido en un tratamiento seguro, eficaz y menos invasivo ${ }^{4}$, pero no es aplicable a casos graves y avanzados como éste.

En este individuo, debido a las perforaciones intestinales, era inminente la realización de una colostomía descompresiva para resolverlas y controlar la infección; no obstante, se presentaron invasión tumoral maligna extensa y adherencias graves de todas las asas intestinales, lo que imposibilitó la creación de estomas convencionales.

Al llevar a cabo una búsqueda exhaustiva en la bibliografía actual no se identificó ningún caso similar en el medio de los autores, donde se realizan estomas artificiales. El caso más semejante lo notificó en 2016 Huang, et al., de una mujer de 72 años con adenocarcinoma seroso y carcinomatosis peritoneal, que sufrió perforación intestinal, pero en quien sí fue posible efectuar una colostomía transversa ${ }^{5}$. Por otra parte Yetisir, et al., informó en 2015 el caso de una paciente de 62 años con distintas afecciones comórbidas: obesidad mórbida, hipertensión, diabetes mellitus, enfermedad pulmonar crónica, antecedente de ocho intervenciones abdominales, entre otros; la paciente se sometió a una operación de emergencia por peritonitis y complicación de hernia gigante y como hallazgos se encontraron adherencias, abdomen séptico, necrosis y perforación de un segmento de íleon de $70 \mathrm{~cm}$ (50 cm proximal a la válvula ileocecal). Se la trató con irrigación abdominal, resección de íleon, anastomosis enteroenteral y cierre abdominal tardío con bolsa de Bogotá. Con posterioridad desarrolló una fístula enteroambiental, tratada con ileostomía, en la cual se utilizó un tubo de drenaje de Pezzer, y tratamiento de presión negativa (tanto en la ileostomía como en el abdomen). Se indicó que la ileostomía maduró en cuatro días, se convirtió a ileostomía en asa y a continuación se instituyó tratamiento de presión negativa intrarrectal sincronizada con la abdominal. Por último, cuatro meses después se cerró la ileostomía por prolapso de estoma y al cuarto día la paciente comenzó a defecar; durante 12 meses de seguimiento no se registraron complicaciones de la ostomía 6 .

Este último caso, al igual que el notificado en este estudio, plantean la posibilidad de nuevos abordajes al realizar estomas en este tipo de pacientes. Debe señalarse que el uso de injertos vasculares de dacrón 
(poliéster) precoagulado con plata e impregnados con rifampicina proporcionan resistencia elevada en infecciones por Staphylococcus aureus resistente a la meticilina y E. coli?.

La paciente de este informe tenía un alto riesgo de perforaciones intestinales, sobre todo por la obstrucción tumoral maligna y también por el riesgo añadido del consumo de bevacizumab, con el cual se ha informado un incremento hasta de tres veces el riesgo de perforación intestinal (que no supera el beneficio terapéutico y por lo cual se decidió emplearlo en esta paciente) ${ }^{8}$. Asimismo, se ha publicado que el $4.2 \%$ de los pacientes atendidos con CCR e HIPEC sufre perforación intestinal (sin relación con anastomosis) ${ }^{9}$; sin embargo, estos procedimientos mejoran los resultados en la calidad de vida e incrementan la sobrevida más de tres años ${ }^{10,11}$.

\section{Conclusiones}

Los estomas autólogos, ileostomías y colostomías han sido la única opción derivativa para el control y tratamiento de diversas enfermedades intestinales; no obstante, en ocasiones no es posible crearlos, por lo que los estomas sintéticos son una buena opción, dado que permiten controlar las infecciones intraabdominales en casos difíciles, como la carcinomatosis peritoneal avanzada. Además, son un recurso importante junto con los tratamientos oncológicos cada vez más eficaces.

\section{Conflicto de intereses}

Los autores declaran que no tienen ningún conflicto de intereses.

\section{Financiamiento}

Este estudio se financió con recursos propios de la institución.

\section{Responsabilidades éticas}

Protección de personas y animales. Los autores declaran que para esta investigación no se han realizado experimentos en seres humanos ni en animales.

Confidencialidad de los datos. Los autores declaran que han seguido los protocolos de su centro de trabajo sobre la publicación de datos de pacientes.

Derecho a la privacidad y consentimiento informado. Los autores han obtenido el consentimiento informado de los pacientes y/o sujetos referidos en el artículo. Este documento obra en poder del autor de correspondencia.

\section{Bibliografía}

1. Correa-Rovelo JM, Vargas RG, Vélez-Pérez FM, Díaz-Girón A. Estomas en coloproctología. En: Morales-Saavedra JL, Torres-Rodríguez VB (eds.). Tratado de cirugía general, vol. II, 3ra ed. Ciudad de México: El Manual Moderno, 2017:1317-1325.

2. Hardy KJ. Surgical history. Evolution of the stoma. Aust N Z J Surg. 1989;59:71-7.

3. Littré A. Quoted in Dinnick T. The origins and evolution of colostomy. Br J Surg 1934;22:142-154

4. Miller ZA, Mohan P, Tartaglione R, Narayanan G. Bowel obstruction: decompressive gastrostomies and cecostomies. Semin Intervent Radiol. 2017;34:349-360.

5. Huang T, Nakamuro M, Nakano H, Okubo Y, Kondo Y, Ishikawa K, et al Successful multidisciplinary treatment of metastatic ovarian cancer after perforative peritonitis of the rectum due to disseminated ovarian cancer. Gan To Kagaku Ryoho. 2016;43:2136-2138.

6. Yetisir F, Salman AE, Acar HZ, Özer M, Aygar M, Osmanoglu G. Management of septic open abdomen in a morbid obese patient with enteroatmospheric fistula by using standard abdominal negative pressure therapy in conjunction with intrarectal one. Case Rep Surg. 2015; 2015:293946

7. Schneider F, O'Connor S, Becquemin JP. Efficacy of collagen silver-coated polyester and rifampin-soaked vascular grafts to resist infection from MRSA and Escherichia coli in a dog model. Ann Vasc Surg 2008;22:815-821.

8. Qi WX, Shen Z, Tang LN, Yao Y. Bevacizumab increases the risk of gastrointestinal perforation in cancer patients: a meta-analysis with a focus on different subgroups. Eur J Clin Pharmacol. 2014;70:893-906.

9. Chouliaras K, Levine EA, Fino N, Shen P, Votanopoulos KI. Prognostic factors and significance of gastrointestinal leak after cytoreductive surgery (CRS) with heated intraperitoneal chemotherapy (HIPEC). Ann Surg Oncol. 2017;24:890-897.

10. Verwaal VJ, Bruin S, Boot H, van Slooten G, van Tinteren H. 8-year follow-up of randomized trial: cytoreduction and hyperthermic intraperitoneal chemotherapy versus systemic chemotherapy in patients with peritoneal carcinomatosis of colorectal cancer. Ann Surg Oncol. 2008; 15:2426-32.

11. Chua TC, Moran BJ, Sugarbaker PH, Levine EA, Glehen O, Gilly FN, Baratti D, et al. Early- and long-term outcome data of patients with pseudomyxoma peritonei from appendiceal origin treated by a strategy of cytoreductive surgery and hyperthermic intraperitoneal chemotherapy. J Clin Oncol. 2012;30:2449-56. 